\title{
A prickly problem: developing a volunteer-friendly tool for monitoring populations of a terrestrial urban mammal, the West European hedgehog (Erinaceus europaeus)
}

\author{
Ben Williams $^{1}$ (D) - Nittanjyot Mann ${ }^{1} \cdot$ Jessica L. Neumann ${ }^{2} \cdot$ Richard W. Yarnell $^{3} \cdot$ Philip J. Baker $^{1}$
}

Published online: 27 August 2018

(C) The Author(s) 2018

\begin{abstract}
Across Europe, hedgehogs (Erinaceus europaeus) appear to be in decline in both urban and rural landscapes. Current methods used to monitor urban populations are, however, associated with several potential limitations. In this study, we conducted hedgehog footprint-tunnel surveys in 219 residential gardens across Reading, UK between May-September in 2013 and/or 2014; gardens were surveyed for five continuous days. Single-species occupancy models were used to investigate factors influencing hedgehog occupancy and two-species occupancy models were used to estimate a species interaction factor (SIF) between hedgehogs and (a) badgers (Meles meles), (b) foxes (Vulpes vulpes) and (c) dogs (Canis familiaris). The five-day survey protocol was associated with a falseabsence error rate of $0.1-0.4 \%$, indicating that it was a reliable method for determining hedgehog presence; conversely, $34.7 \%$ of householders were not able to correctly predict hedgehog presence or absence. Hedgehogs were widely distributed across Reading, but detected in only 32-40\% of gardens. None of the within-garden or outside-garden factors investigated significantly affected hedgehog occupancy in the single-species models, but the two-species models indicated that badgers (SIF $=0.471 \pm 0.188$ ), but not foxes $(\mathrm{SIF}=0.954 \pm 0.048)$ or dogs $(\mathrm{SIF}=0.780 \pm 0.228)$, negatively affected the presence of hedgehogs in gardens, although not significantly. Overall, footprint-tunnels represent a viable field method for monitoring urban hedgehog populations, however, other approaches are required to identify factors that make gardens "hedgehog friendly".
\end{abstract}

Keywords Citizen science $\cdot$ Erinaceus europaeus $\cdot$ Hedgehog $\cdot$ Population monitoring $\cdot$ Urban conservation $\cdot$ Urban wildlife

Ben Williams

b.williams@pgr.reading.ac.uk; bensmailliw@gmail.com

Nittanjyot Mann

n.mann@ student.reading.ac.uk

Jessica L. Neumann

j.1.neumann@reading.ac.uk

Richard W. Yarnell

richard.yarnell@ntu.ac.uk

Philip J. Baker

p.j.baker@ reading.ac.uk

1 School of Biological Sciences, University of Reading, Whiteknights, Reading, Berkshire RG6 6AS, UK

2120 Russell Building, Department of Geography and Environmental Science, University of Reading, Whiteknights, Reading, Berkshire RG6 6AB, UK

3 School of Animal, Rural and Environmental Sciences, Nottingham Trent University, Brackenhurst, Southwell, Nottinghamshire NG25 OFQ, UK

\section{Introduction}

Urbanisation is one of the most significant forms of habitat modification undertaken by humans, typically resulting in marked changes in e.g. animal behaviour, reproductive output, community composition and nutrient cycling (Marzluff et al. 2008; Gaston 2010; Douglas et al. 2011; Niemelä 2011; Forman 2014). In many instances, the construction of urban areas leads to species' declines or extirpations, although some "urban adapters" may thrive in such modified landscapes (Blair 1996; Kettel et al. 2018). Indeed, for some species, urban areas may represent a refuge habitat in which they may be able to escape some of the biotic and abiotic pressures present in other habitats and/or which offer new opportunities to be exploited (Bateman and Fleming 2012).

The West European hedgehog (Erinaceus europaeus) is a medium-sized $(<1.2 \mathrm{~kg})$ insectivorous mammal found from Spain and Italy north to Scandinavia (Morris and Reeve 2008). In many parts of its range the species is thought to be in decline (Huijser and Bergers 2000; van de Poel et al. 2015); in the UK, 
data from several monitoring schemes indicate that populations have declined markedly since the 1950s (Harris et al. 1995; Battersby 2005; Wembridge 2011; Roos et al. 2012) leading to its designation as a species of conservation concern in 2007 (Joint Nature Conservation Committee 2010). Possible reasons for this decline include: changes associated with agricultural intensification such as the loss of hedgerows, increased field sizes and reductions in the availability of invertebrate prey (Krebs et al. 1999; Hof and Bright 2010, 2012; Haigh et al. 2012; Hof et al. 2012; Moorhouse et al. 2014); the increased abundance of Eurasian badgers (Meles meles) (Judge et al. 2014, 2017), an intra-guild predator (Doncaster 1994); an increase in the density of road networks and associated traffic (Becher and Griffiths 1998; Rondinini and Doncaster 2002); and climate mediated effects on food availability and over-winter hibernation (Jackson 2007; Morrs 2018). Conversely, other studies (Young et al. 2006; Hubert et al. 2011; Parrott et al. 2014; Trewby et al. 2014; van de Poel et al. 2015; Williams et al. 2018) have indicated an increased tendency for hedgehogs to be found within, or associated with, areas dominated by human habitation, including towns and cities (Hof and Bright 2009).

From a conservation perspective, urban areas pose a number of challenges. Potentially the biggest problem, but simultaneously the biggest opportunity, is that the major habitat present is residential gardens. Although gardens collectively cover a large area (21-27\% of UK cities: Loram et al. 2007; Davies et al. 2009), each one is typically small $\left(\sim 190 \mathrm{~m}^{2}\right.$ : Davies et al. 2009), privately-owned and has to deliver a range of functions (Cameron et al. 2012). As such, garden-based conservation strategies have to persuade large numbers of householders to put aside areas of their property for the benefit of wildlife (Goddard et al. 2010), although such actions are not always successful (Gaston et al. 2005; Matteson and Langellotto 2011). Identifying factors within a garden that can help promote focal species is, therefore, a priority.

Monitoring wildlife populations in urban areas is also typically reliant on engaging substantial numbers of urban residents because of the fact that wild animals cannot often be observed easily from publicly accessible areas. Previous studies aimed at monitoring hedgehog populations in urban areas in the UK have relied extensively on questionnaire surveys where participants are typically asked whether they believe hedgehogs are present in their garden and/or sightings of hedgehogs in gardens or other urban habitats (Toms and Newson 2006; Baker and Harris 2007; Hof and Bright 2009). Although the use of volunteers can help reduce costs whilst simultaneously increasing statistical power and helping communities engage with conservation issues (Toms and Newson 2006; Schmeller et al. 2009; Silvertown 2009; Mackechnie et al. 2011; Wright et al. 2013), citizen science based approaches can be associated with limitations which reduce their reliability as a mechanism for guiding management actions (e.g. Perkins et al. 2013). For example, strategies used to recruit volunteers can generate biased samples (e.g. participants may be more likely to submit positive records: sensu Scott et al. 2014), participants may vary in their ability to identify different species (Dickinson et al. 2010) and species such as hedgehogs may exhibit behavioural patterns that make them elusive (Dowding et al. 2010) such that householders may not always be aware that the focal species is present in their garden (Williams et al. 2014). The latter would lead to an increased frequency of "false absences" (i.e. failing to record a species when it is present). Consequently, there is the need for a method which can be used by householders to record hedgehog presence reliably.

One potentially suitable method is footprint-tunnels (Huijser and Bergers 2000; Yarnell et al. 2014; Williams et al. 2018); these are designed to document hedgehog presence by using food bait to lure individuals across an ink pad so that their distinctive footprints are recorded. The major advantages of this approach are: (i) they are cheap (unit price for the current study was approximately $£ 5$ ); (ii) they can record hedgehog activity remotely throughout the $24 \mathrm{~h}$ cycle; (iii) footprint papers can be returned to researchers for verification; and (iv) they can be used easily by volunteers. For example, the use of these tunnels has been illustrated on several UK television programmes and, as a result, individual householders are known to have purchased and successfully used their own simply out of personal interest. In addition, this method can be easily incorporated into an occupancy analysis framework (MacKenzie et al. 2006; Yarnell et al. 2014), a maximum likelihood technique used specifically to estimate presence/absence whilst accounting for imperfect detection. Such models can also incorporate covariates to identify factors affecting a species' distribution (MacKenzie et al. 2006); these could then be used as the basis for advice to householders about making their gardens more hedgehog friendly.

Therefore, in this study, we conducted a survey of residential gardens in Reading, UK using footprint-tunnels deployed by volunteer householders according to a standardised protocol with the specific objectives of: (i) identifying a suitable survey protocol as a method for the future monitoring of hedgehogs in urban landscapes; and (ii) identifying factors associated with the presence/absence of hedgehogs in residential gardens.

\section{Materials and methods}

The study was conducted in Reading, UK $\left(51^{\circ}, 27^{\prime} \mathrm{N}: 0^{\circ}, 58^{\prime}\right.$ W) during May-September 2013-2014 inclusive. Reading is a large town which straddles the River Thames and covers an area of approximately $55 \mathrm{~km}^{2}$; the human population is approximately 230,000 people (Fig. 1). There are four major residential sectors within the town surrounding the town's central commercial district: Caversham (North), Woodley 


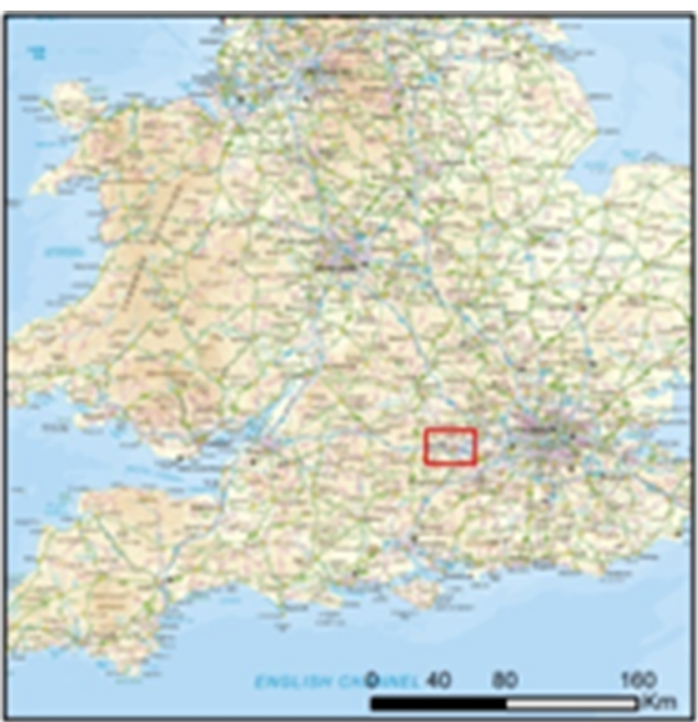

$-1$

\section{Legend}

- Houses surveyed in 2013

- Houses surveyed in 2014

Houses surveyed in both years

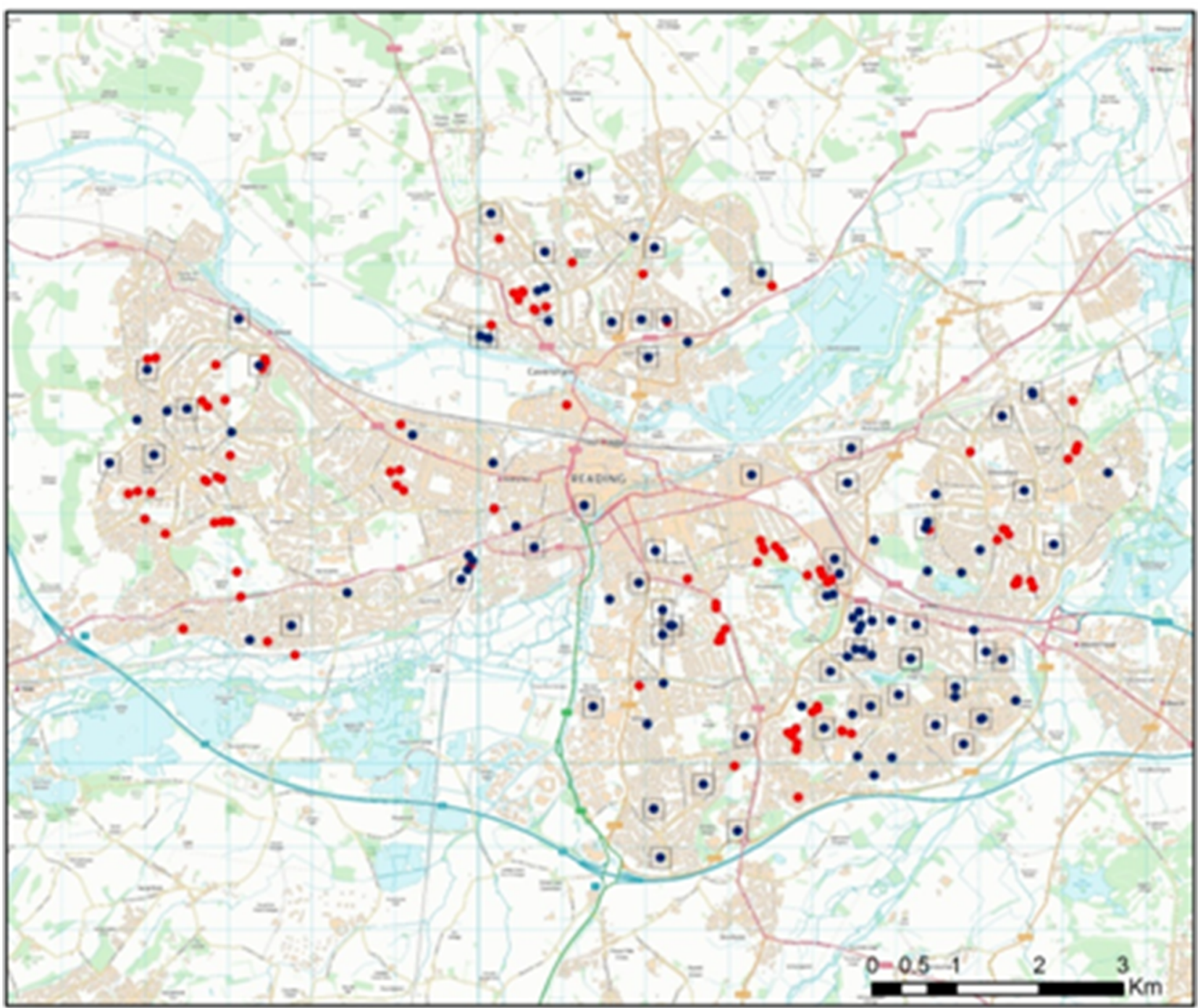

Ordnance Survey Crown Copyright 2014. All Rights Reserved.

Fig. 1 Distribution of gardens surveyed for hedgehogs in Reading, UK, in 2013 and/or 2014 
(East), Earley (South) and Tilehurst (West). Individual gardens were surveyed in one or both years.

To ensure coverage across the city as a whole, and to engage volunteers who were likely to have and not have hedgehogs in their gardens, a pseudo-random recruitment protocol was used. Within each 1-km Ordnance Survey grid square $(N=55)$, two $500 \mathrm{~m} \times 500 \mathrm{~m}$ quadrants were selected at random: leaflets were then delivered to $c .50$ houses in the centre of each of these quadrants. Leaflets specifically requested that householders volunteer to take part in the study regardless of whether they thought hedgehogs did or did not visit their garden, but that they thought hedgehogs could potentially access their garden via holes under fences or gates and/or via gaps in boundaries.

Gardens were surveyed using triangular footprint-tunnels constructed from corrugated plastic (Correx $\AA$ ) measuring $1200 \mathrm{~mm}$ in length, $210 \mathrm{~mm}$ wide and $180 \mathrm{~mm}$ high (Yarnell et al. 2014). The base contained a removable insert onto which a piece of A4 paper $(297 \mathrm{~mm} \times 210 \mathrm{~mm})$ was attached at each end. A petri dish was placed at the centre of the base insert to house the food bait (commercially available dry Spikes ${ }^{\circledR}$ hedgehog food). Ink made from carbon powder mixed with vegetable oil was applied to two strips of masking tape between the food and each piece of paper.

Approximately 20-30 gardens were surveyed at any one time, with equipment recycled between volunteers so that successive batches (groups of gardens surveyed over the same five night period) were investigated. Surveyors from each of the four major residential sectors were included in each batch to ensure that any spatial differences in detection rates were not confounded with the time of surveying. Volunteers in close proximity to one another were allocated to separate batches for surveying.

Each householder was given one footprint-tunnel to mimic the likely pattern of surveying achievable by persons who may opt to purchase their own. Householders were instructed to place the tunnel in their rear garden in a position where they thought hedgehogs would be likely to encounter it (e.g. parallel to fences at points where animals could enter the garden). Surveys were conducted in rear gardens as hedgehogs are known to avoid front gardens (Dowding et al. 2010). Each garden was surveyed for five continuous days, with the tunnel checked every morning. If footprints (of any species) were present and/or if the food bait had been taken, the paper and/ or bait were replaced respectively. All footprint papers were returned for verification by the authors. Each night was treated as a repeat survey and gardens were classified as occupied (hedgehog-positive gardens) if hedgehog footprints were recorded on any of the five nights.

Chi-squared tests were used to quantify: (a) the consistency of hedgehog presence/absence in gardens between years for that subset of gardens surveyed in both 2013 and $2014(N=60)$; and (b) the relationship between the householders' predictions that hedgehogs would/would not be detected and the actual pattern of detection ( $N=147$ gardens surveyed in 2014).

\section{Footprint-tunnels as a monitoring tool}

Data on the five-day pattern of presence-absence of hedgehogs in each garden were analysed using occupancy analysis (MacKenzie et al. 2006). We assessed the goodness of fit for the most global modal using a bootstrap method (100 replications) resulting in a variance inflation factor of $\hat{c}=2.08$. Therefore, Akaike's Information Criterion (AIC) values were modified by the variance inflation factor $(\hat{c})$ to give quasi-AIC (QAIC) values for use in subsequent model selection procedures (Anderson and Burnham 2002). Models with $\triangle$ QAIC values $>2$ or which did not converge were excluded as having little or no support (Burnham and Anderson 2002); standard errors were inflated by a factor of $\sqrt{\hat{c}}=1.44$.

Initial analyses compared two baseline models independently of any covariates and which assumed that daily detection rates were (i) constant or (ii) variable; the optimal model was selected based on the minimum QAIC value. Models were constructed separately for: houses surveyed in (a) 2013 and (b) 2014; and (c) using the last available data from each household (i.e. data from 2013 for houses surveyed only in 2013 and 2014 for those surveyed in both years or in 2014 only: hereafter "pooled" data). Data were analysed using PRESENCE v12.7 (Hines 2006). Naïve occupancy rate is defined as the proportion of gardens surveyed where hedgehogs were detected (the latter are hereafter termed "hedgehog-positive gardens"); the true occupancy rate is estimated by accounting for false-absences.

The suitability of the survey protocol for the future monitoring of hedgehogs in residential gardens in the UK was assessed by estimating the number of sites needed to detect $(\alpha=0.05) 50 \%, 25 \%$ and $10 \%$ changes in occupancy between two surveys with $2-5$ days of surveying per garden at 0.80 , 0.90 and 0.95 levels of power. Estimates of occupancy and detection were derived from the pooled data. Analyses were conducted in R (Anonymous 2008) using code provided by Guillera-Arroita and Lahoz-Monfort (2012): power was calculated as the proportion of 5000 simulations in which a significant difference was detected.

\section{Factors affecting hedgehog occupancy}

Factors within gardens that could potentially affect the presence of hedgehogs were quantified using a questionnaire survey of participants. Questionnaires requested information on: house type (HOUSETYPE: detached, semi-detached, other), as this is related to the size of the garden (Loram et al. 2008) and, to some extent, access down the side of the house; the percentage aerial cover of lawn (BACKLAWN), flowerbeds (BACKFLOWER) and shrubs (BACKSHRUB) in the rear garden; whether the rear 
garden contained a pond/other water feature (WATER), a compost heap (COMPOST) or log pile (LOGS); whether householders thought hedgehogs could access their back garden from their front garden (FRONT2BACK); if foxes (Vulpes vulpes) (FOX) and/or badgers (BADGER) visited their garden (at least yearly); if they owned a dog (Canis familiaris) (DOG); whether any supplementary food from feeding either foxes, hedgehogs, badgers and/or birds on the ground was available at least once a month (FOOD); and whether they used slug pellets (SLUGPELLET), weed killer (WEEDKILLER), rat or mouse poison (POISON) or chemical fertilisers (FERTILISER). Percentage coverage was converted to standardised $\mathrm{Z}$ values as recommended by Donovan and Hines (2007). POISON and FERTILISER were subsequently omitted from all analyses as too few householders stated that they used these compounds. DOG was also omitted from these single species occupancy models because only 16 residents reported owning a dog in the subset of data ( $N=132$ households) analysed. However, it was included in a further two-species occupancy model (see below) because sample sizes were slightly larger.

Models also included parameters summarising the garden's location within the town (DISTRICT: i.e. which of the four major residential sectors it was located in) and four metrics for habitats outside the garden: the distance to the edge of the town (EDGE); and nearest allotment gardens (ALLOTMENT), amenity grassland (e.g. park, sports field, school playing field: AMENITY) and woodland (WOODLAND). Distances were determined using ArcMAP v10.1 based on Ordnance Survey 1:10,000 maps checked against Google Maps satellite layer (Google Maps 2015).

Models included no more than one covariate for occupancy and detection due to limited samples sizes; models were fitted with a constant daily detection rate, as this was shown to better fit the data than models with variable daily detection rates (see Results). Model fit was assessed using the bootstrap procedure in PRESENCE (MacKenzie and Bailey 2004). This is a Monte-Carlo type simulation process in which the detection and occupancy rates identified by the original model are used to randomly assign sites as occupied or unoccupied for each of 100 simulations: a Pearson chi-squared statistic is then generated for each run and compared to the original observed $\chi^{2}$ value; the model is considered to fit the data well if the observed value falls within the range calculated across the simulation process. The significance of individual covariates was determined by whether the corresponding $95 \%$ confidence interval crossed zero or not.

\section{Associations with predators and domestic dogs}

Fisher's exact tests and two-species occupancy models were used to investigate the likelihood of hedgehogs co-occurring in gardens with badgers, foxes or dogs (MacKenzie et al. 2004, 2006). The latter estimates a species interaction factor
(SIF) which is a ratio of the likelihood of two species cooccurring compared to a hypothesis of independence: a value $<1$ indicates avoidance (i.e. the two species co-occur less frequently than would be expected if they were distributed independently) whereas a value $>1$ indicates aggregation (i.e. the two species co-occur more frequently than would be expected if they were distributed independently) (e.g. Luiselli 2006; Bailey et al. 2009). As two-species occupancy models tend not to converge when covariates are added, they were left out of the models (Richmond et al. 2010).

\section{Results}

Overall, 219 gardens were surveyed: 51 in 2013 only, 108 in 2014 only and 60 in both years (Fig. 1). Naïve occupancy rates for those gardens surveyed in 2013 and 2014 were $31.5 \%(N=111)$ and $39.9 \%(N=168)$, respectively (Table 1). The pattern of detection of hedgehogs in gardens surveyed in both years $(N=60)$ was highly consistent $\left(\chi^{2}{ }_{1}=\right.$ $17.631, p<0.001)$ with 31 households $(51.7 \%)$ failing to record hedgehogs in either year, $16(26.7 \%)$ recording hedgehogs in both years and $5(8.3 \%)$ and $8(13.3 \%)$ households recording hedgehogs only in the first or second year of the study, respectively.

There was a significant association between predicted patterns of occupancy in gardens based on householders' perceptions and the actual detection of hedgehogs in gardens $\left(\chi^{2}{ }_{1}=\right.$ $14.529, p<0.001)$. Overall, $52(35.4 \%)$ and $44(29.9 \%)$ householders correctly predicted the absence and presence of hedgehogs, respectively. However, hedgehogs were recorded in $19(12.9 \%)$ gardens where householders thought they were absent, and were not recorded in 32 (21.8\%) gardens where householders thought they were present. Collectively, these data indicate an error rate of $34.7 \%$.

\section{Footprint-tunnels as a monitoring tool}

On average, hedgehogs were detected in approximately 60 $65 \%$ of hedgehog-positive gardens on a night-by-night basis (Fig. 2a). There was an apparent difference in the cumulative pattern of detection of hedgehogs between the two years, with $>90 \%$ of positive gardens identified after two days of surveying in 2013 but only after four days in 2014 (Fig. 2b). Similarly, a higher proportion of hedgehog-positive gardens were visited on all five nights in 2013, whereas a higher proportion of hedgehog-positive gardens were visited on just one night in 2014 (Fig. 2c). Collectively, hedgehogs were recorded on $21.6 \%$ and $24.3 \%$ of tunnel-nights in 2013 ( $N=555$ tunnel-nights) and $2014(N=840)$, respectively; comparable figures considering hedgehog-positive gardens only were $68.6 \%$ ( $N=175$ tunnel-nights) and 60.9\% $(N=335)$ (Fig. 2c). On 
Table 1 Summary of models to estimate the probability of occupancy $(\Psi( \pm \mathrm{SE}))$ of hedgehogs in residential gardens in Reading based upon constant (two parameters) versus variable (six parameters) daily detection rates

\begin{tabular}{|c|c|c|c|c|c|c|c|c|c|c|}
\hline \multirow[t]{2}{*}{ Year } & \multirow[t]{2}{*}{ Model } & \multirow[t]{2}{*}{ QAIC } & \multirow[t]{2}{*}{$\triangle \mathrm{QAIC}$} & \multirow[t]{2}{*}{ AIC weight } & \multirow[t]{2}{*}{ Model likelihood } & \multirow[t]{2}{*}{ Naïve $\Psi$} & \multirow{2}{*}{$\begin{array}{l}\text { Estimated } \Psi \\
( \pm \mathrm{SE})\end{array}$} & \multirow{2}{*}{$\begin{array}{l}\text { Detection rate } \\
( \pm \mathrm{SE})\end{array}$} & \multicolumn{2}{|c|}{ No. of surveys needed $( \pm 1.96 \mathrm{SE})$} \\
\hline & & & & & & & & & $\begin{array}{l}80 \% \text { confidence } \\
\text { interval }\end{array}$ & $\begin{array}{l}95 \% \text { confidence } \\
\text { interval }\end{array}$ \\
\hline 2013 & $\begin{array}{l}\text { Constant } \\
\text { Variable }\end{array}$ & $\begin{array}{l}121.36 \\
128.73\end{array}$ & $\begin{array}{l}0.00 \\
7.37\end{array}$ & $\begin{array}{l}0.9755 \\
0.0245\end{array}$ & $\begin{array}{l}1.0000 \\
0.0251\end{array}$ & 0.315 & $0.316(0.077)$ & $0.684(0.062)$ & $1.4(1.0-2.0)$ & $2.6(1.8-3.6)$ \\
\hline 2014 & $\begin{array}{l}\text { Constant } \\
\text { Variable }\end{array}$ & $\begin{array}{l}210.62 \\
215.24\end{array}$ & $\begin{array}{l}0.00 \\
4.62\end{array}$ & $\begin{array}{l}0.9097 \\
0.0903\end{array}$ & $\begin{array}{l}1.0000 \\
0.0993\end{array}$ & 0.399 & $0.403(0.069)$ & $0.603(0.050)$ & $1.7(1.3-2.3)$ & $3.2(2.5-4.3)$ \\
\hline Pooled & $\begin{array}{l}\text { Constant } \\
\text { Variable }\end{array}$ & $\begin{array}{l}228.43 \\
234.00\end{array}$ & $\begin{array}{l}0.00 \\
5.57\end{array}$ & $\begin{array}{l}0.9419 \\
0.0581\end{array}$ & $\begin{array}{l}1.0000 \\
0.0617\end{array}$ & 0.370 & $0.373(0.063)$ & $0.615(0.048)$ & $1.7(1.3-2.2)$ & $3.1(2.4-4.1)$ \\
\hline
\end{tabular}

Results are given for houses surveyed in $2013(\mathrm{~N}=111)$ and $2014(\mathrm{~N}=168)$. Naïve occupancy is the proportion of sites surveyed where hedgehogs were detected: estimated occupancy is the proportion of gardens estimated to have hedgehogs after correcting for false-absences; estimated occupancy rates are given only for constant detection rate models. Detection rate indicates the probability of detecting hedgehogs in gardens where they were present on any given day of the 5-day survey period. The number of surveys needed is the number of consecutive survey nights required to be $80 \%$ and $95 \%$ confident that non-detection reflects the true absence of hedgehogs. Since the number of surveys needed is derived from a sample estimate of detection, the confidence intervals around the number of sites needed were calculated from the SEs derived from the detection estimates (McArdle 1990). $\triangle$ QAIC is the difference in QAIC value between each model and the current top-ranked model (that with the lowest QAIC). AIC weight is a measure of support for each model being the 'best' model
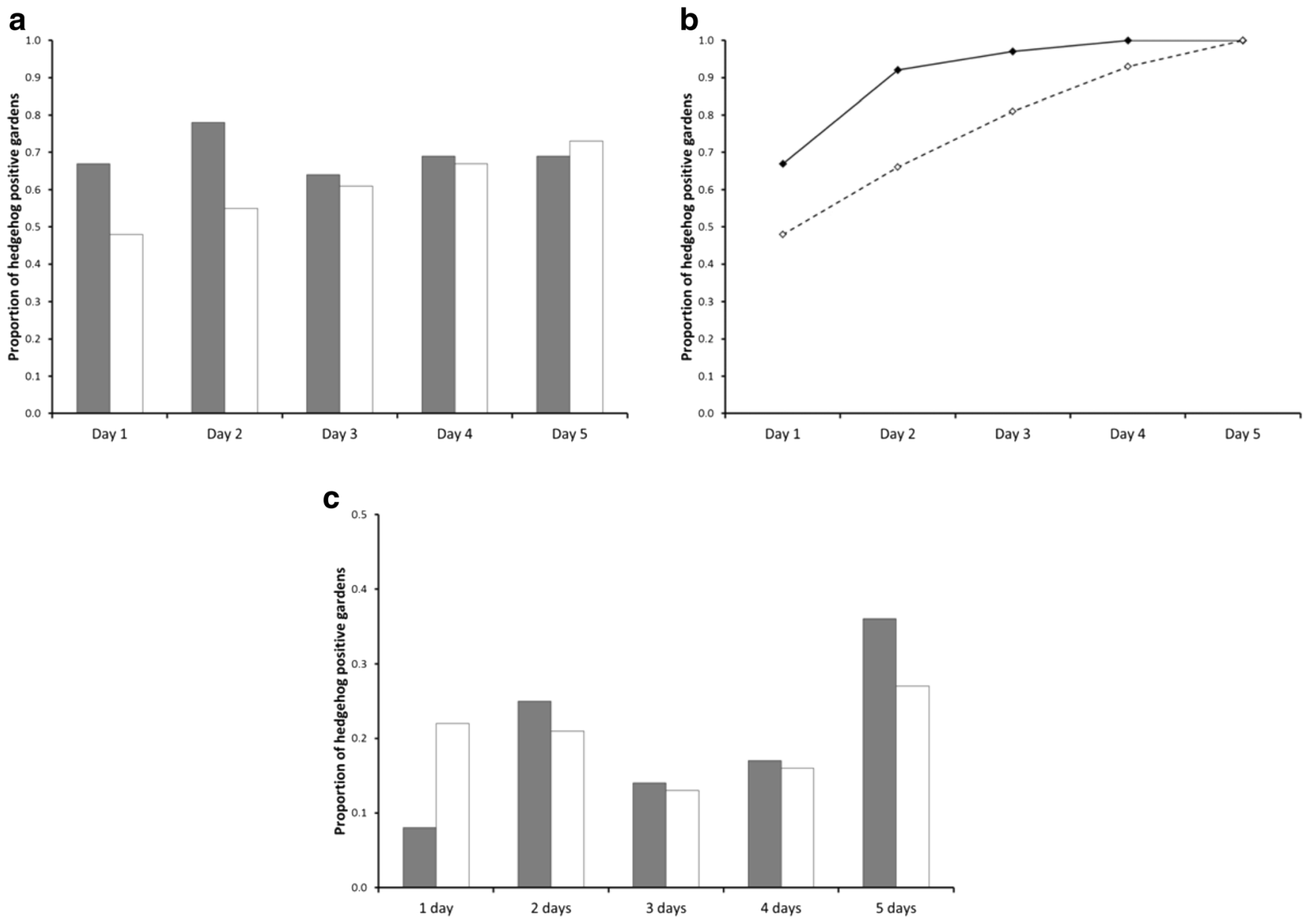

Fig. 2 Summary of: (a) the proportion of hedgehog-positive gardens where hedgehogs were detected each day; (b) the cumulative pattern of detection of hedgehogs in hedgehog-positive gardens; and (c) the number of days where hedgehogs were detected in each hedgehog-positive garden. Shaded columns/solid lines and open columns/dotted lines denote hedgehog-positive gardens surveyed in $2013(\mathrm{~N}=36)$ and $2014(N=67)$ respectively 
average, hedgehog-positive gardens were visited on 3.2 nights.

Despite these differences, however, occupancy models with a constant daily detection rate performed better than those with survey-specific detection rates for both years (Table 1). Power analyses indicated that approximately 3.1 nights of surveying were required to be $95 \%$ confident of detecting hedgehogs (Table 1). Consequently, differences between the naïve and estimated occupancy rates were very small: false-absence error rates were $0.1 \%$ in 2013 and $0.4 \%$ in 2014.

Sample sizes required to detect different levels of change to different levels of statistical power are outlined in Table 2. In comparison with those sample sizes achieved in different field studies of terrestrial mammals in the UK (badger: $N=1614$ 2578 (Cresswell et al. 1989; Wilson et al. 1997; Judge et al. 2014); brown hare (Lepus europaeus): $N=751$ (Hutchings and Harris 1996); hedgehog: $N=111-1925$ (Toms and Newson 2006; Roos et al. 2012; Yarnell et al. 2014; Williams et al. 2018); red fox: $N=160-444$ (Baker et al. 2002; Webbon et al. 2004); see also Battersby 2005), this survey protocol would be suitable for detecting changes in the order of $25 \%$ with $95 \%$ power at a national level $(N=$ 668 gardens).

\section{Factors affecting hedgehog occupancy}

Overall, 151 (68.9\%) participants returned their questionnaire. Of these, 132 (60.3\% of all householders) were complete and used for the occupancy analysis. Only two models had $\triangle$ QAIC values $<2$ (Table 3). However, based on $95 \%$ confidence intervals, none of the variables considered significantly affected hedgehog occupancy, although, two did affect hedgehog detection (FOOD, FRONT2BACK).

\section{Associations with predators and domestic dogs}

Of the 151 gardens for which data were available from the questionnaire survey, hedgehogs, badgers and foxes visited 64 (42.4\%), $36(23.8 \%)$ and $51(33.7 \%)$ gardens, respectively; dogs were present in 22 (14.6\%) gardens. Collectively, badgers, dogs and/or foxes visited or were present in 133 (88.1\%) gardens.

There was no significant difference in the relative numbers of gardens where hedgehogs were detected in relation to the presence/absence of badgers (Fisher's exact test: $p=0.123$ ), foxes $(p=0.605)$ or dogs $(p=0.352)$. Hedgehogs were detected in $46.1 \%$ of gardens where badgers were absent $(N=115)$ and $30.6 \%$ of gardens where badgers were present $(N=36)$ : comparable figures for gardens visited by foxes were $44.0 \%$ $(N=100)$ and $39.2 \%(N=51)$; and for gardens where dogs were present, $44.2 \%(N=129)$ and $31.8 \%(N=22)$. However, the SIF between badgers and hedgehogs was $0.471 \pm 0.188$ indicating that hedgehogs were less likely to co-occur with badgers than would be expected under an independence hypothesis, although this was not significant $(95 \%$ CI: $-1.538,0.030)$. Conversely, the SIF values between hedgehogs and foxes $(0.954 \pm 0.048)$ and hedgehogs and dogs $(0.780 \pm 0.228)$ indicate that hedgehogs co-occurred with both these species as would be expected under an independence hypothesis.

\section{Discussion}

Previous studies aimed at documenting the use of gardens by hedgehogs in the UK have focussed on questionnaire surveys (Baker and Harris 2007) and timed or anecdotal observations (Toms and Newson 2006; Hof and Bright 2009, 2016; Wembridge and Langton 2016). The current study suggests that such approaches may be problematic. In this study, $34.7 \%$
Table 2 Results of power analysis showing the number of residential gardens that would need to be surveyed to detect a significant percentage change in site occupancy by hedgehogs in relation to survey effort (no. of days each garden surveyed) and different levels of statistical power

\begin{tabular}{lllll}
\hline \% change in occupancy & Survey effort (no. of days surveyed) & \multicolumn{2}{l}{$\begin{array}{l}\text { No. of sites required to achieve stated level } \\
\text { of statistical power }\end{array}$} \\
\cline { 2 - 4 } & & 0.80 & 0.90 & 0.95 \\
\hline 10 & 2 & 4190 & 5609 & 6937 \\
25 & & 631 & 845 & 1044 \\
50 & 3 & 138 & 185 & 229 \\
10 & & 2954 & 3954 & 4890 \\
25 & & 449 & 601 & 743 \\
50 & 4 & 99 & 133 & 164 \\
10 & & 2716 & 3636 & 4496 \\
25 & & 414 & 554 & 685 \\
50 & 5 & 92 & 123 & 152 \\
10 & & 2647 & 3544 & 4383 \\
25 & & 404 & 540 & 668 \\
50 & & 90 & 120 & 148 \\
\hline
\end{tabular}


Table 3 Summary of occupancy models investigating factors associated with the presence/ absence of hedgehogs in residential gardens $(\mathrm{N}=132)$ in Reading, UK

\begin{tabular}{|c|c|c|c|c|c|}
\hline Model & QAIC & $\Delta \mathrm{QAIC}$ & AIC wgt & $\begin{array}{l}\text { Model } \\
\text { likelihood }\end{array}$ & $\begin{array}{l}\text { Number of } \\
\text { parameters }\end{array}$ \\
\hline$\Psi$ (BADGER),p (FOOD) & 258.97 & 0.00 & 0.3469 & 1.0000 & 4 \\
\hline$\Psi(),. \mathrm{p}(\mathrm{FOOD})$ & 259.56 & 0.59 & 0.2583 & 0.7445 & 3 \\
\hline$\Psi$ (BADGER),p (FRONT2BACK) & 262.08 & 3.11 & 0.0733 & 0.2112 & 4 \\
\hline$\Psi(),. \mathrm{p}($ FRONT2BACK) & 262.63 & 3.66 & 0.0556 & 0.1604 & 3 \\
\hline$\Psi$ (BADGER),p (FOX) & 264.46 & 5.49 & 0.0223 & 0.0642 & 4 \\
\hline$\Psi(),. \mathrm{p}(\mathrm{FOX})$ & 264.98 & 6.01 & 0.0172 & 0.0495 & 3 \\
\hline$\Psi$ (BADGER),p(.) & 265.04 & 6.07 & 0.0167 & 0.0481 & 3 \\
\hline$\Psi($ COMPOST),p(.) & 265.17 & 6.20 & 0.0156 & 0.0450 & 3 \\
\hline$\Psi(),. \mathrm{p}()$. & 265.59 & 6.62 & 0.0127 & 0.0365 & 2 \\
\hline$\Psi$ (FRONT2BACK),p(.) & 265.80 & 6.83 & 0.0114 & 0.0329 & 3 \\
\hline$\Psi($ LOGS),p(.) & 266.40 & 7.43 & 0.0084 & 0.0244 & 3 \\
\hline$\Psi(),. \mathrm{p}$ (ALLOTMENT) & 266.49 & 7.52 & 0.0081 & 0.0233 & 3 \\
\hline$\Psi(),. \mathrm{p}(\mathrm{COMPOST})$ & 266.51 & 7.54 & 0.0080 & 0.0231 & 3 \\
\hline$\Psi(\mathrm{FOX}), \mathrm{p}()$. & 266.70 & 7.73 & 0.0073 & 0.0210 & 3 \\
\hline$\Psi(),. \mathrm{p}$ (BACKFLOWER) & 266.80 & 7.83 & 0.0069 & 0.0199 & 3 \\
\hline$\Psi$ (HOUSETYPE),p(.) & 266.81 & 7.84 & 0.0069 & 0.0198 & 3 \\
\hline$\Psi(),. \mathrm{p}$ (AMENITY) & 266.84 & 7.87 & 0.0068 & 0.0195 & 3 \\
\hline$\Psi$ (BACKLAWN),p(.) & 267.04 & 8.07 & 0.0061 & 0.0177 & 3 \\
\hline$\Psi(\mathrm{AMENITY}), \mathrm{p}()$. & 267.05 & 8.08 & 0.0061 & 0.0176 & 3 \\
\hline$\Psi(),. \mathrm{p}$ (HOUSETYPE) & 267.13 & 8.16 & 0.0059 & 0.0169 & 3 \\
\hline$\Psi(),. \mathrm{p}(\mathrm{LOGS})$ & 267.22 & 8.25 & 0.0056 & 0.0162 & 3 \\
\hline$\Psi(),. \mathrm{p}$ (BADGER) & 267.25 & 8.28 & 0.0055 & 0.0159 & 3 \\
\hline$\Psi$ (WEEDKILLER),p(.) & 267.28 & 8.31 & 0.0054 & 0.0157 & 3 \\
\hline$\Psi(),. \mathrm{p}$ (SLUGPELLET) & 267.33 & 8.36 & 0.0053 & 0.0153 & 3 \\
\hline$\Psi(),. \mathrm{p}(\mathrm{EDGE})$ & 267.38 & 8.41 & 0.0052 & 0.0149 & 3 \\
\hline$\Psi($ FOOD),p(.) & 267.38 & 8.41 & 0.0052 & 0.0149 & 3 \\
\hline$\Psi(),. \mathrm{p}$ (WOODLAND) & 267.39 & 8.42 & 0.0052 & 0.0148 & 3 \\
\hline$\Psi(),. \mathrm{p}$ (WATER) & 267.41 & 8.44 & 0.0051 & 0.0147 & 3 \\
\hline$\Psi$ (ALLOTMENT),p(.) & 267.46 & 8.49 & 0.0050 & 0.0143 & 3 \\
\hline$\Psi$ (BACKSHRUB),p(.) & 267.49 & 8.52 & 0.0049 & 0.0141 & 3 \\
\hline$\Psi$ (SLUGPELLET),p(.) & 267.50 & 8.53 & 0.0049 & 0.0141 & 3 \\
\hline$\Psi$ (EDGE),p(.) & 267.52 & 8.55 & 0.0048 & 0.0139 & 3 \\
\hline$\Psi$ (BACKFLOWER),p(.) & 267.54 & 8.57 & 0.0048 & 0.0138 & 3 \\
\hline$\Psi(),. \mathrm{p}$ (WEEDKILLER) & 267.58 & 8.61 & 0.0047 & 0.0135 & 3 \\
\hline$\Psi(),. \mathrm{p}$ (BACKSHRUB) & 267.58 & 8.61 & 0.0047 & 0.0135 & 3 \\
\hline$\Psi(),. \mathrm{p}(\mathrm{BACKLAWN})$ & 267.58 & 8.61 & 0.0047 & 0.0135 & 3 \\
\hline$\Psi$ (WOODLAND),p(.) & 267.58 & 8.61 & 0.0047 & 0.0135 & 3 \\
\hline$\Psi(),. \mathrm{p}$ (DISTRICT) & 267.59 & 8.62 & 0.0047 & 0.0134 & 3 \\
\hline$\Psi$ (WATER),p(.) & 267.59 & 8.62 & 0.0047 & 0.0134 & 3 \\
\hline$\Psi$ (DISTRICT),p(.) & 267.59 & 8.62 & 0.0047 & 0.0134 & 3 \\
\hline
\end{tabular}

Models were selected on the basis of Quasi-Akaike's Information Criterion (QAIC) values. Models with $\triangle$ QAIC values $>2$ or which did not converge were excluded as having little or no support (Burnham and Anderson 2002). Variables are described in the text of 147 householders failed to correctly predict the presence or absence of hedgehogs across the 5-day sampling period. Similarly, in their footprint-tunnel study in Gloucestershire, UK, Williams et al. (2014) reported that hedgehogs were only recorded in $35 \%$ of gardens $(N=23)$ where householders had reported seeing them previously, but were also recorded in $38 \%$ of gardens $(N=24)$ where the householder had not reported seeing them. Although houses were studied for only five days in each study, and householders may be basing their perceptions on longer time frames, both studies suggest that a large proportion of householders may be unaware of the pattern of use of their gardens by hedgehogs.

Footprint-tunnels do, however, appear to offer a clear solution to this problem; the 5-day sampling period used in this study was associated with a false-absence error rate of just
$0.1-0.4 \%$. In addition, the technique is self-evidently one that can be applied easily by members of the general public, as they collected all the data used in the current analyses. Based on the current study, the 5-day sampling protocol used would be capable of detecting changes in the order of $25 \%$ with $95 \%$ power with moderate levels of citizen participation $(N=668$ gardens). In actuality, given the hedgehog's wide appeal to members of the public, it is not unreasonable to assume that much larger sample sizes would be achievable. For example, the Hogwatch survey (Hof and Bright 2016) received information from $>16,000$ people; such high levels of participation suggest that much smaller changes in urban hedgehog numbers could be detected using this methodology. However, it is worth noting that hedgehog-positive gardens in Reading were visited on almost twice as many nights (mean of 3.2) as those 
in Gloucestershire (1.7 nights: Williams et al. 2014). The reason for this disparity is not clear, but such variations would affect the methodology's statistical power.

\section{Factors affecting the use of gardens by hedgehogs}

Hedgehogs were found in 32-40\% of gardens in Reading across the two years of the study, a figure very similar to the $36 \%$ reported by Williams et al. (2014) in Gloucestershire. Therefore, although hedgehogs appear to be widely distributed within individual urban areas (hedgehogs were detected in all four residential sectors within Reading, with no significant differences in occupancy), they appear to be utilising only a minority of gardens. This is potentially worrying as the occupancy rates identified in this study are likely to be maximum levels, since the recruitment protocol was, if anything, likely to engage people with hedgehogs in their garden (even though we specifically asked for volunteers not to base their involvement on an any prior knowledge of hedgehogs in their garden).

Alternatively, this does indicate that urban areas might be capable of holding much higher densities of hedgehogs than they do currently, if those factors that make gardens "hedgehog-friendly" could be identified. However, none of the within-garden or outside-garden habitat factors incorporated into single-species occupancy models significantly affected hedgehog occupancy (see also Williams et al. 2014): the presence of badgers was the only factor included in the two highest-ranked models, but this was not significant.

There are several potential explanations for these results. For example, it may be that the factors included in these models did not reflect the characteristics actually selected for by hedgehogs. However, this is unlikely. The variables selected represent a range of important biotic processes (the availability of food and shelter, predation risk, inter-specific competition, habitat connectivity and distance to more natural habitats), some of which have been shown to affect rural and urban hedgehogs in other studies (Young et al. 2006; Hubert et al. 2011; Parrott et al. 2014; Trewby et al. 2014; van de Poel et al. 2015).

Alternatively, this lack of difference may reflect the fact that back gardens represent multi-functional space (Cameron et al. 2012), such that ground-level microhabitats likely to be important to hedgehogs are often overtly similar at the neighbourhood level (Loram et al. 2008) with a strong emphasis on cultivated lawns, (non-native) flower borders (Smith et al. 2006) and features such as sheds, decking and patios. One factor that does affect garden structure and habitat richness, however, is garden size (Loram et al. 2008), with larger gardens containing a broader range of micro-habitats. This pattern is, in turn, evident between house types, with larger gardens historically being associated with detached houses and the smallest gardens with terraced houses, although this pattern is changing; the increased emphasis on high-density, low-cost housing throughout the UK means that garden size is becoming more similar across a broad range of house types. Within this study, however, occupancy was not affected by house type nor by the residential sector where the garden was located, suggesting that garden size does not, per se, affect their suitability for hedgehogs.

However, footprint-tunnels are associated with one significant limitation in the context of discriminating between gardens. As they are baited with relatively small amounts of food bait in an attempt to minimise their impact on normal patterns of hedgehog movement, they are likely to be visited by animals regardless of whether the tunnel is positioned within a garden where the animal spends a great deal of its time foraging versus one where the animal may simply be travelling. Consequently, tunnel visits may not always reflect "good" gardens. Therefore, future studies will require other field methods, such as radio- or GPS-tracking (e.g. Glasby and Yarnell 2013), to identify characteristics associated with the differential use of individual gardens.

Despite this limitation, the two-species occupancy modelling did identify that the use of gardens by hedgehogs is potentially influenced by the presence of badgers, although this result was not significant; the presence of foxes had no observable effect. Both badgers and foxes represent potential predators and competitors of hedgehogs (Pettett et al. 2018) and declines in the abundance of both badgers (due to culling to manage bovine tuberculosis) and foxes (due to an outbreak of sarcoptic mange) have been associated with increases in hedgehogs in rural (Trewby et al. 2014) and urban (Harris and Baker 2000) habitats, respectively. Similarly, Pettett et al. (2018) reported a negative relationship between hedgehogs and both badgers and foxes based on sightings of animals killed on roads. As such, hedgehogs might be expected to avoid using gardens frequented by these larger species.

Evidence for this is, however, equivocal. For example, Ward et al. (1997) documented only a short-term avoidance (5-30 min) of badger odour by hedgehogs. Furthermore, urban areas contain large amounts of natural foods and food supplied deliberately by humans, often targeted at focal species such as badgers, foxes and/or hedgehogs (e.g. Baker et al. 2000; Bateman and Fleming 2012). These anthropogenic foods may, therefore, act to reduce competition by increasing the volume of food available, but also minimising the risk of predation since predators are likely to be well-fed and foods supplied by householders require minimal foraging effort compared to having to break through the defences of a curled hedgehog. In addition, conservation NGOs also recommend that householders supply food for hedgehogs in covered feeding stations for protection but which also reduces the likelihood of food being stolen by other species. Supplying food in this way could lead to spatial convergence and temporal divergence of hedgehog foraging patterns relative to those of the 
other two species, thereby favouring co-existence. As such, reported "increases" in hedgehogs in relation to declines in badgers or foxes may well represent increases in abundance, but also changes in avoidance-related movement patterns; identifying which mechanism(s) are involved would require studies focussing on both simultaneous patterns of movement and population demographics.

Domestic pets may also pose a threat to hedgehogs, particularly in urban areas where their density is very high (Baker et al. 2010). For example, of 19,618 hedgehogs admitted to the Royal Society for the Prevention of Cruelty to Animals (RSPCA) from 2006 to 2016 inclusive, 592 (3\%) individuals were reported as having been attacked by another animal; 51 $(0.3 \%)$ were specifically listed as having been attacked by a dog and $5(<0.1 \%)$ by a cat (A. Grogan, pers. comm.). The impact of either species on hedgehog occupancy is, however, likely to be minimal, principally because most pet dogs are kept indoors for at least part of the night, the time when hedgehogs are active. In addition, there is an abundance of anecdotal observations (e.g. internet videos and authors' personal observations) where, having been disturbed by a pet dog, a hedgehog continues to forage after the dog has been recalled by the owner or it loses interest. Unsurprisingly, therefore, the species interaction factor for hedgehogs and dogs calculated in this study $(0.780 \pm 0.228)$ suggests that the two species cooccur independently of one another. But it is worth noting that the proportion of householders in this study that owned dogs (14.6\%) is much lower than the $30-31 \%$ reported for the nation as a whole (Murray et al. 2010, 2015). One potential explanation for this difference could be that householders with dogs were less likely to volunteer for this study because they perceived hedgehogs were not likely to be present in their garden, even though we emphasized that people should participate even if they thought hedgehogs did not visit their garden. Therefore, although are data suggest that dog ownership does not influence hedgehog occupancy, further investigation would be prudent.

Unlike pet dogs, pet cats are often allowed to roam freely at night in the UK (Thomas et al. 2012, 2014). Given their density and agility, they are ubiquitous within the urban environment, with virtually all gardens being frequented at one time or another. As such, an occupancy based approach would not be able to distinguish any avoidance patterns by hedgehogs, if it were to exist. However, it is not likely that hedgehogs would avoid gardens where cats are present since they pose little direct threat and, again, multiple records exist of the two species in the same garden at the same time.

That being said, juvenile hedgehogs would be more vulnerable to both cats and dogs, as well as foxes and badgers, such that they may be expected to show greater levels of avoidance of both wild and domestic predators than adult animals. This is particularly important in the context of factors that might limit population recruitment in urban hedgehog populations. Consequently, studies on factors affecting the ranging behaviour of juvenile hedgehogs would be beneficial.

In summary, this study has demonstrated that footprinttunnels represent an effective "citizen science" technique for monitoring urban hedgehog populations and which overcome the potential problems associated with sightings-based techniques. In addition, they are cheap (the $£ 5$ cost mentioned could be further reduced by getting householders to build a similar design using materials that they are likely to have lying around) and the data collected can be easily verified, either by returning or photographing footprint papers. The data presented here, and elsewhere, suggest that hedgehogs can typically be found throughout the urban landscape, but may only be utilising a minority $(\leq 40 \%)$ of gardens: although this is concerning, it does imply that substantive improvements could be made. Therefore, more detailed studies are urgently required to identify those within- and outside-garden factors that influence garden use by hedgehogs.

Acknowledgements We would like to thank the many Reading householders who participated in this study and Karl Schroepfer, Scott Hudson, Sarah Murton and Sarah Putnam who helped with surveying. The project was conducted in collaboration with the People's Trust for Endangered Species and the British Hedgehog Preservation Society.

Open Access This article is distributed under the terms of the Creative Commons Attribution 4.0 International License (http:// creativecommons.org/licenses/by/4.0/), which permits unrestricted use, distribution, and reproduction in any medium, provided you give appropriate credit to the original author(s) and the source, provide a link to the Creative Commons license, and indicate if changes were made.

\section{References}

Anderson DR, Burnham KP (2002) Avoiding pitfalls when using information-theoretic methods. J Wildlife Manage 66:912-918

Anonymous (2008) A language and environment for statistical computing. In: R Foundation for statistical computing. Vienna, Austria

Bailey LL, Reid JA, Forsman ED, Nichols JD (2009) Modeling cooccurrence of northern spotted and barred owls: accounting for detection probability differences. Biol Conserv 142:2983-2989

Baker PJ, Funk SM, Harris S, White PCL (2000) Flexible spatial organization of urban foxes, Vulpes vulpes, before and during an outbreak of sarcoptic mange. Anim Behav 59:127-146

Baker PJ, Harris S, Webbon CC (2002) Effect of British hunting ban on fox numbers. Nature 419:34

Baker PJ, Harris S (2007) Urban mammals: what does the future hold? An analysis of the factors affecting patterns of use of residential gardens in Britain. Mammal Rev 37:297-315

Baker PJ, Soulsbury CD, Iossa G, Harris S (2010) Domestic cat (Felis catus) and domestic dog (Canis familiaris). In: Gehrt SD, Riley SPD, Cypher BL (eds) Urban carnivores. Ecology, conflict, and conservation. John Hopkins University Press, Baltimore

Bateman PW, Fleming PA (2012) Big city life: carnivores in urban environments. J Zool 287:1-23

Battersby, J., (2005) UK Mammals: Species Status and Population Trends. First Report by the Tracking Mammals Partnership. Joint 
Nature Conservation Committee/Tracking Mammals Partnership, Peterborough

Becher SA, Griffiths R (1998) Genetic differentiation among local populations of the European hedgehog (Erinaceus europaeus) in mosaic habitats. Mol Ecol 7:1599-1604

Blair RB (1996) Land use and avian species diversity along an urban gradient. Ecol Appl 6:506-519

Burnham KP, Anderson DR (2002) Model selection and multi-model inference. Springer, Berlin; Germany

Cameron RWF, Blanuša T, Taylor JE, Salisbury A, Halstead AJ, Henricot B, Thompson K (2012) The domestic garden - its contribution to urban green infrastructure. Urban For Urban Green 11:129-137

Cresswell P, Harris S, Bunce RGH, Jefferies DJ (1989) The badger (Meles meles) in Britain: present status and future population changes. Biol J Linn Soc 38:91-101

Davies ZG, Fuller RA, Loram A, Irvine KN, Sims V, Gaston KJ (2009) A national scale inventory of resource provision for biodiversity within domestic gardens. Biol Conserv 142:761-771

Dickinson JL, Zuckerberg B, Bonter DN (2010) Citizen science as an ecological research tool: challenges and benefits. Annu Rev Ecol Evol Syst 41:149-172

Doncaster CP (1994) Factors regulating local variations in abundance: field tests on hedgehogs, Erinaceus europaeus. Oikos 69:182-192

Donovan M, Hines, JE (2007) Exercises in Occupancy Estimation and Modelling. Retrieved from: http://www.uvm.edu/envnr/vtcfwru/ spreadsheets/occupancy.htm. [Accessed: 9th June 2016]

Douglas I, Goode D, Houck MC, Wang R (2011) The Routledge handbook of urban ecology. Routledge, London

Dowding CV, Harris S, Poulton S, Baker PJ (2010) Nocturnal ranging behaviour of urban hedgehogs, Erinaceus europaeus, in relation to risk and reward. Anim Behav 80:13-21

Forman RTT (2014) Urban ecology. Science of cities. Cambridge University Press, Cambridge

Gaston KJ (2010) Urban ecology. Cambridge University Press, Cambridge

Gaston KJ, Smith RM, Thompson K, Warren PH (2005) Urban domestic gardens (II): experimental tests of methods for increasing biodiversity. Biodivers Conserv 14:395-413

Glasby L, Yarnell RW (2013) Evaluation of the performance and accuracy of global positioning system bug transmitters deployed on a small mammal. Eur J Wildl Res 59:915-919

Goddard MA, Dougill AJ, Benton TG (2010) Scaling up from gardens: biodiversity conservation in urban environments. Trends Ecol Evol 25:90-98

Google Maps (2015) Reading, UK. Satellite. Retrieved from https://www. google.com/maps/place/Reading,+UK/@51.4447709,-0. $9864359,10563 \mathrm{~m} / \mathrm{data}=! 3 \mathrm{~m} 1 ! 1 \mathrm{e} 3 ! 4 \mathrm{~m} 2 ! 3 \mathrm{~m} 1 ! 1 \mathrm{~s} 0 \times 48742078 \mathrm{~d} 93 \mathrm{~d} 3 \mathrm{db} 7$ : 0x2ae19f7fcefa7994 [Accessed 3 June 2015]

Guillera-Arroita G, Lahoz-Monfort JJ (2012) Designing studies to detect differences in species occupancy: power analysis under imperfect detection. Methods Ecol Evol 3:860-869

Haigh A, Butler F, O'Riordan M (2012) Intra- and inter-habitat differences in hedgehog distribution and potential prey availability. Mammalia 76:261-268

Harris S, Baker P (2000) Urban foxes, Second edn. Whittet Books, Essex

Harris S, Morris P, Wray S, Yalden D (1995) A review of British mammals: population estimates and conservation status of British mammals other than cetaceans. Joint Nature Conservation Committee, Peterborough

Hines JE (2006) PRESENCE 5.3 - Software to estimate patch occupancy and related parameters. USGS-PWRC

Hof AR, Bright PW (2009) The value of green-spaces in built-up areas for western hedgehogs. Lutra 52:69-82

Hof AR, Bright PW (2010) The value of Agri-environment schemes for macro-invertebrate feeders: hedgehogs on arable farms in Britain. Anim Conserv 13:467-473
Hof AR, Bright PW (2012) Factors affecting hedgehog presence on farmland as assessed by questionnaires. Acta Theriol 57:79-88

Hof AR, Bright PW (2016) Quantifying the long-term decline of the west European hedgehog in England by subsampling citizen-science datasets. Eur J Wildl Res 62:407-413

Hof AR, Snellenberg J, Bright PW (2012) Food or fear? Predation risk mediates edge refuging in an insectivorous mammal. Anim Behav 83:1099-1106

Hubert P, Julliard R, Biagianti S, Poulle M-L (2011) Ecological factors driving the higher hedgehog (Erinaceus europaeus) density in an urban area compared to the adjacent rural area. Landscape Urban Plan 103:34-43

Huijser MP, Bergers PJM (2000) The effects of roads and traffic on hedgehog (Erinaceus europaeus) populations. Biol Conserv 95: 111-116

Hutchings MR, Harris S (1996) The current status of the brown hare (Lepus europaeus) in Britain, Joint Nature Conservation Committee, Peterborough

Jackson DB (2007) Factors affecting the abundance of introduced hedgehogs (Erinaceus europaeus) to the Hebridean island of south Uist in the absence of natural predators and implications for nesting birds. J Zool 271:210-217

Joint Nature Conservation Committee (2010) UK Priority Species pages: Erinaceus europaeus. Available at: http://jncc.defra.gov.uk/ speciespages/2253.pdf [Accessed 4 December 2015]

Judge J, Wilson GJ, Macarthur R, Delahay RJ, McDonald RA (2014) Density and abundance of badger social groups in England and Wales 2011-2013. Sci Rep 4:3809

Judge J, Wilson GJ, Macarthur R, McDonald RA, Delahay RJ (2017) Abundance of badgers (Meles meles) in England and Wales. Sci Rep $7: 276$

Kettel EF, Gentle LK, Quinn JL, Yarnell RW (2018) The breeding performance of raptors in urban landscapes: a review and meta-analysis. J Ornithol 159:1-18

Krebs JR, Wilson JD, Bradbury RB, Siriwardena GM (1999) The second silent spring? Nature 400:611-612

Loram A, Tratalos J, Warren PH, Gaston KJ (2007) Urban domestic gardens $(\mathrm{X})$ : the extent and structure of the resource in five major cities. Landsc Ecol 22:601-615

Loram A, Warren PH, Gaston KJ (2008) Urban domestic gardens (XIV): the characteristics of gardens in five cities. Environ Manag 42:361376

Luiselli L (2006) Site occupancy and density of sympatric Gaboon viper (Bitis gabonica) and nose-horned viper (Bitis nasicornis). J Trop Ecol 22:555-564

Mackechnie C, Maskell L, Norton L, Roy D (2011) The role of 'big society' in monitoring the state of the environment. J Environ Monitor 13:2687-2691

MacKenzie DI, Bailey LL (2004) Assessing the fit of site-occupancy models. J Agr Biol Envir St 9:300-318

MacKenzie DI, Bailey LL, Nichols JD (2004) Investigating species cooccurrence patterns when species are detected imperfectly. J Animal Ecol 73:546-555

MacKenzie DI, Nichols JD, Royle JA, Pollock KH, Bailey LL, Hines JE (2006) Occupancy estimation and modelling: inferring patterns and dynamics of species occurrence. Academic Press, London

Marzluff JM, Shulenberger E, Endlicher W, Alberti M, Bradley G, Ryan C, Simon U, ZumBrunnen C (2008) Urban ecology. An international perspective on the interaction between humans and nature. Springer, New York

Matteson KC, Langellotto GA (2011) Small scale additions of native plants fail to increase beneficial insect richness in urban gardens. Insect Conserv Diver 4:89-98

McArdle BH (1990) When are rare species not there? Oikos 57:276-277 
Moorhouse TP, Palmer SCF, Travis JMJ, Macdonald DW (2014) Hugging the hedges: might Agri-environment manipulations affect landscape permeability for hedgehogs? Biol Conserv 176:109-116

Morrs P (2018) Hedgehogs. William Collins, London

Morris PA, Reeve NJ (2008) Hedgehog Erinaceus europaeus. In: Harris S, Yalden DW (Eds) Mammals of the British Isles: handbook, $4^{\text {th }}$ edition. The Mammal Society, Southampton

Murray JK, Browne WJ, Roberts MA, Whitmarsh A, Gruffydd-Jones TJ (2010) Number and ownership profiles of cats and dogs in the UK. Vet Record 166:163-168

Murray JK, Gruffydd-Jones TJ, Roberts MA, Browne WJ (2015) Assessing changes in the UK pet cat and dog populations: numbers and household ownership. Vet Record 177:259

Niemelä J (2011) Urban ecology. Patterns, processes, and applications. Oxford University Press, Oxford

Parrott D, Etherington TR, Dendy J (2014) A geographically extensive survey of hedgehogs (Erinaceus europaeus) in England. Eur J Wildl Res 60:399-403

Perkins G, Kutt A, Vanderduys E, Perry J (2013) Evaluating the costs and sampling adequacy of a vertebrate monitoring program. Aust Zool $36: 373-380$

Pettett CE, Johnson PJ, Moorhouse TP, Macdonald DW (2018) National predictors of hedgehog Erinaceus europaeus distribution and decline in Britain. Mammal Rev 48:1-6

Richmond OMW, Hines JE, Beissinger SR (2010) Two-species occupancy models: a new parameterization applied to co-occurrence of secretive rails. Ecol Appl 20:2036-2046

Rondinini C, Doncaster CP (2002) Roads as barriers to movement for hedgehogs. Funct Ecol 16:504-509

Roos S, Johnston A, Noble D (2012) UK hedgehog datasets and their potential for long-term monitoring. BTO Research Report No. 598, British Trust for Ornithology, Thetford, Norfolk, UK

Schmeller DS, Henry PY, Julliard R, Gruber B, Clobert J, Dziock F, Lengyel S, Nowicki P, Deri E, Budrys E, Kull T, Tali K, Bauch B, Settele J, Van Swaay C, Kobler A, Babij V, Papastergiadou E, Henle $\mathrm{H}$ (2009) The advantages of volunteer-based biodiversity monitoring in Europe. Conserv Biol 23:307-316

Silvertown J (2009) A new dawn for citizen science. Trends Ecol Evol 24: $467-470$

Scott DM, Berg MJ, Tolhurst BA, Chauvenet ALM, Smith GC, Neaves K, Lochhead J, Baker PJ (2014) Changes in the distribution of red foxes (Vulpes vulpes) in urban areas in Great Britain: findings and limitations of a media-driven nationwide survey. PLoS One 9: e99059

Smith RM, Thompson K, Hodgson JG, Warren PH, Gaston KJ (2006) Urban domestic gardens (IX): composition and richness of the vascular plant flora, and implications for native biodiversity. Biol Conserv 129:312-322

Thomas RL, Fellowes MDE, Baker PJ (2012) Spatio-temporal variation in predation by urban domestic cats (Felis catus) and the acceptability of possible management actions in the UK. PLoS One 7:e49369

Thomas RL, Baker PJ, Fellowes MDE (2014) Ranging characteristics of the domestic cat (Felis catus) in an urban environment. Urban Ecosyst 17:911-921

Toms MP, Newson SE (2006) Volunteer surveys as a means of inferring trends in garden mammal populations. Mammal Rev 36:309-317

Trewby ID, Young R, McDonald RA, Wilson GJ, Davison J, Walker N, Robertson A, Doncaster CP, Delahay RJ (2014) Impacts of removing badgers on localised counts of hedgehogs. PLoS One 9:e95477

van de Poel JL, Dekker J, van Langevelde F (2015) Dutch hedgehogs Erinaceus europaeus are nowadays mainly found in urban areas, possibly due to the negative effects of badgers Meles meles. Wildlife Biol 21:51-55

Ward JF, Macdonald DW, Doncaster CP (1997) Responses of foraging hedgehogs to badger odour. Anim Behav 53:709-720

Webbon CC, Baker PJ, Harris S (2004) Faecal density counts for monitoring changes in red fox numbers in rural Britain. J Appl Ecol 41: 768-779

Wembridge D (2011) The state of Britain's hedgehogs. People's Trust for Endangered Species, London

Wembridge D, Langton S (2016) Living with mammals: an urban study. British Wildl 27:188-195

Williams B, Baker P, Thomas E, Wilson G, Judge J, Yarnell RW (2018) Reduced occupancy of hedgehogs (Erinaceus europaeus) in rural England and Wales: the influence of habitat and an asymmetric intra-guild predator. Sci Rep

Williams RL, Stafford R, Goodenough AE (2014) Biodiversity in urban gardens: assessing the accuracy of citizen science data on garden hedgehogs. Urban Ecosyst 18:819-833

Wilson G, Harris S, McLaren G (1997) Changes in the British badger population, 1988-1997. People's Trust for Endangered Species, London

Wright LJ, Newson SE, Noble DG (2013) The value of a random sampling design for annual monitoring of national populations of larger British mammals. Eur J Wildl Res 60:213-221

Yarnell RW, Pacheco M, Williams B, Neumann JL, Rymer DJ, Baker PJ (2014) Using occupancy analysis to validate the use of footprinttunnels as a method for monitoring the hedgehog Erinaceus europaeus. Mammal Rev 44:234-238

Young RP, Davison J, Trewby ID, Wilson GJ, Delahay RJ, Doncaster CP (2006) Abundance of hedgehogs (Erinaceus europaeus) in relation to the density and abundance of badgers (Meles meles). J Zool 269: 349-356 